Swarthmore College

Works

$10-1-2010$

\title{
Three-Dimensional Reconnection And Relaxation Of Merging Spheromak Plasmas
}

Timothy G. Gray , '01

Vyacheslav S. Lukin , '00

Michael R. Brown

Swarthmore College, doc@swarthmore.edu

C. D. Cothran

Follow this and additional works at: https://works.swarthmore.edu/fac-physics

Part of the Physics Commons

Let us know how access to these works benefits you

\section{Recommended Citation}

Timothy G. Gray , '01; Vyacheslav S. Lukin , '00; Michael R. Brown; and C. D. Cothran. (2010). "ThreeDimensional Reconnection And Relaxation Of Merging Spheromak Plasmas". Physics Of Plasmas.

Volume 17, Issue 10. DOI: 10.1063/1.3492726

https://works.swarthmore.edu/fac-physics/108

This work is brought to you for free by Swarthmore College Libraries' Works. It has been accepted for inclusion in Physics \& Astronomy Faculty Works by an authorized administrator of Works. For more information, please contact myworks@swarthmore.edu. 


\section{AIP | Physics of \\ Plasmas}

\section{Three-dimensional reconnection and relaxation of merging spheromak plasmas}

T. Gray, V. S. Lukin, M. R. Brown, and C. D. Cothran

Citation: Physics of Plasmas (1994-present) 17, 102106 (2010); doi: 10.1063/1.3492726

View online: http://dx.doi.org/10.1063/1.3492726

View Table of Contents: http://scitation.aip.org/content/aip/journal/pop/17/10?ver=pdfcov

Published by the AIP Publishing

\section{Articles you may be interested in}

Bulk ion acceleration and particle heating during magnetic reconnection in a laboratory plasmaa)

Phys. Plasmas 21, 055706 (2014); 10.1063/1.4874331

The influence of intense electric fields on three-dimensional asymmetric magnetic reconnection Phys. Plasmas 20, 061204 (2013); 10.1063/1.4811123

Spontaneous three-dimensional magnetic reconnection in merging toroidal plasma experiment Phys. Plasmas 20, 012106 (2013); 10.1063/1.4774403

Publisher's Note: "Three-dimensional reconnection and relaxation of merging spheromak plasmas" [Phys.

Plasmas17, 102106 (2010)]

Phys. Plasmas 17, 129901 (2010); 10.1063/1.3524299

Magnetohydrodynamic study of three-dimensional instability of the spontaneous fast magnetic reconnection Phys. Plasmas 16, 052903 (2009); 10.1063/1.3095562

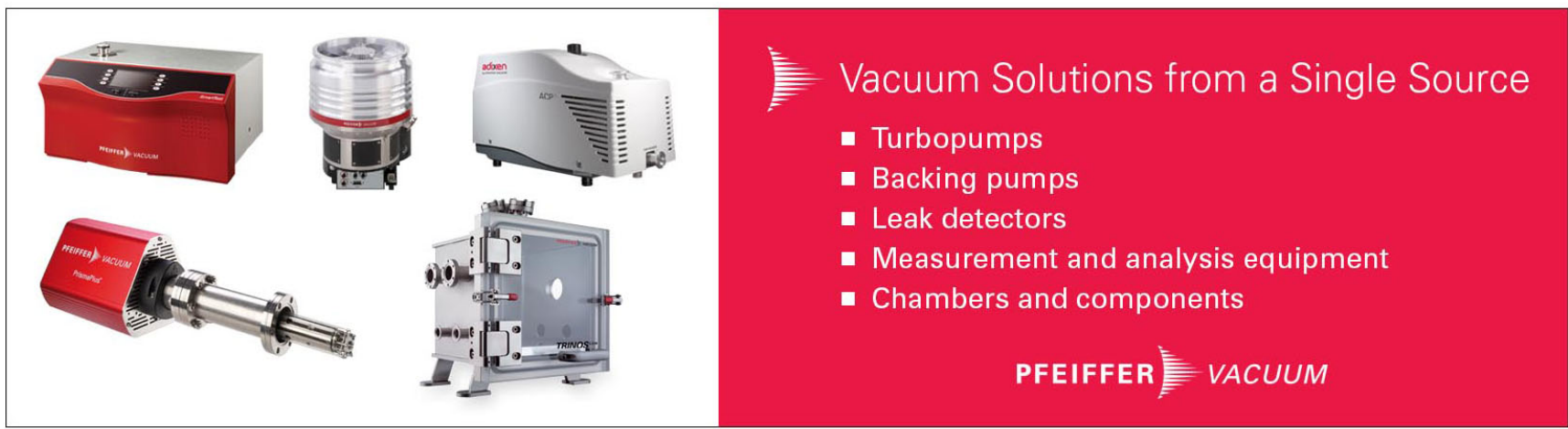




\title{
Three-dimensional reconnection and relaxation of merging spheromak plasmas
}

\author{
T. Gray, ${ }^{1, a)}$ V. S. Lukin, ${ }^{2}$ M. R. Brown, ${ }^{1}$ and C. D. Cothran ${ }^{1, b)}$ \\ ${ }^{1}$ Department of Physics and Astronomy, Swarthmore College, Swarthmore, Pennsylvania 19081-1397, USA \\ ${ }^{2}$ Space Science Division, Naval Research Laboratory, Washington, DC 20375, USA
}

(Received 3 June 2010; accepted 3 September 2010; published online 21 October 2010; corrected 3 November 2010)

\begin{abstract}
Plasma relaxation inside a highly conducting cylindrical boundary is studied both experimentally and computationally. Dynamics are initiated by the introduction of two equal helicity spheromaks at either end of the cylinder. In the experiment, dense, high-magnetic-flux spheromaks are injected into the flux conserving volume with magnetized plasma guns. In the simulation, identical spheromaks initially occupy both halves of the cylinder and a perturbation is introduced. Merging commences with a single three-dimensional null-point that moves radially out of the flux conserving volume at velocities up to 0.2 of the reconnection outflow velocity. Relaxation to the minimum energy state occurs in about ten Alfvén times. An important conclusion is that even though the dynamical activity is limited to a few modes, this activity is sufficient to promote relaxation to the final, minimum energy state. The dynamical activity appears to conserve magnetic helicity while magnetic energy is converted to flow and heat. The final state arrived at dynamically is identical to that described by C. D. Cothran et al. [Phys. Rev. Lett. 103, 215002 (2009)] using static, eigenvalue analysis. (C) 2010 American Institute of Physics. [doi:10.1063/1.3492726]
\end{abstract}

\section{INTRODUCTION}

Spheromaks ${ }^{1}$ are compact, high $\beta$, translatable plasma configurations with a simply connected boundary typically formed in a close-fitting flux-conserver with a single source. ${ }^{2-5}$ Merging of spheromaks was developed by Ono ${ }^{6}$ and further studied at the Swarthmore Spheromak Experiment $(\mathrm{SSX}){ }^{7,8}$ In the past, these experiments have merged spheromaks of opposite helicity (one right-handed, the other left-handed) in order to form a field-reversed configuration and to study magnetic reconnection. This paper describes the process of cohelicity merging of spheromaks (spheromaks with the same handedness).

A joint study consisting of a cohelicity merging experiment performed in SSX and an idealized simulation conducted with a three-dimensional (3D) implicit spectral element HiFi code ${ }^{9}$ is presented in this paper. In both cases, the initial state consists of two spheromaks of the same handedness. Merging of two spheromaks with the same handedness is termed "cohelicity merging." The toroidal fields of the injected spheromaks are oppositely directed while the poloidal fields are codirected. In the interface layer between the two spheromaks, the magnetic field is purely radial and directed inward everywhere with a magnetic field null-point $(\boldsymbol{B}=0)$ located at the center of the volume. This is in distinction from the TS-3 experiments, ${ }^{10}$ where poloidal fields in the interface layer were counterdirected. The system ultimately relaxes to the minimum energy state subject to the constraint of maintaining the helicity of the two injected spheromaks.

Magnetic reconnection in laboratory and astrophysical,

\footnotetext{
${ }^{a)}$ Electronic mail: tgray1@swarthmore.edu.

${ }^{b)}$ Present address: Global Strategies Group, Crofton, MD 21114.
}

or idealized computational settings, has been widely investigated in the past decade. ${ }^{11} \mathrm{~A}$ common driver for magnetic reconnection is the relaxation and self-organization of magnetized plasmas. Woltjer-Taylor theory ${ }^{12-14}$ states that magnetized plasmas relax toward the lowest available energy state characterized by

$$
\nabla \times \boldsymbol{B}=\lambda \boldsymbol{B},
$$

where $\lambda$ is constant. The system minimizes total magnetic energy

$$
W=\frac{1}{2 \mu_{0}} \int \boldsymbol{B}^{2} d v,
$$

constrained by the total magnetic helicity

$$
K=\int \boldsymbol{A} \cdot \boldsymbol{B} d v
$$

where helicity can be interpreted as the linkage of magnetic flux with magnetic flux. Woltjer showed that force-free states with constant $\lambda$ [solutions to Eq. (1)] are the lowest energy state for a closed system. ${ }^{14}$ Given an appropriate dissipation mechanism, magnetic energy would decay faster than magnetic helicity, providing a means for a magnetic system to relax to this preferred final state of minimum energy. Taylor stated that provided a finite resistivity, magnetic reconnection conserves global helicity and provides for the dissipation mechanism for reducing magnetic energy. ${ }^{12,13}$ According to the theory, dissipation occurs at microscopic scales, causing the rate of magnetic energy decay to be much larger than the helicity decay rate. It is also easy to show that solutions of Eq. (1) satisfy 


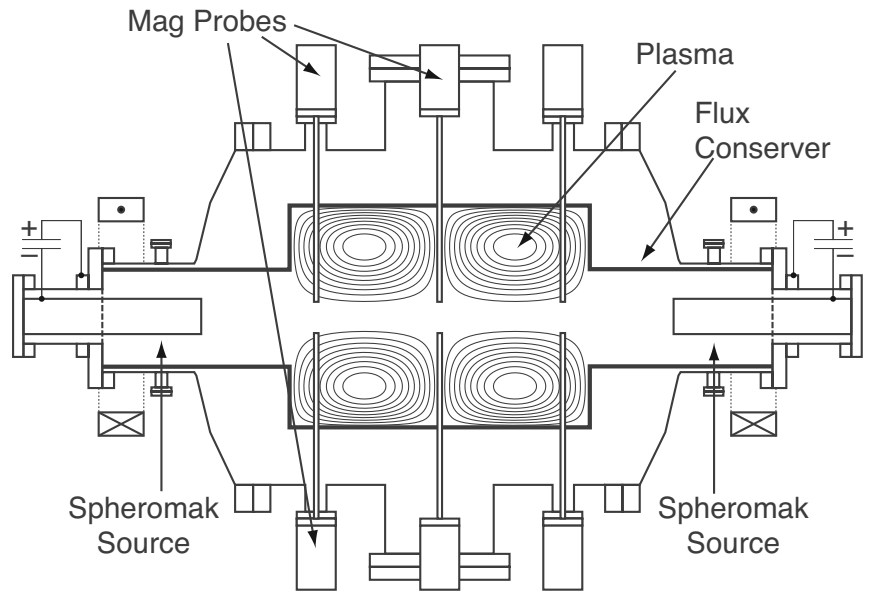

FIG. 1. A cross section of the SSX device. The spheromaks are injected from the coaxial magnetized plasma guns from either end of the machine into the flux-conserver. The whole machine possesses cylindrical symmetry. The flux-conserver is illustrated by the dark line. Representative flux surfaces of the two initial spheromaks are also shown.

$$
\lambda=2 \mu_{0} \frac{W}{K},
$$

thereby relating $\lambda$, total magnetic energy $W$, and total magnetic helicity $K$ of a minimum energy state.

While spheromak experiments typically have axisymmetric configurations, not all geometries have lowest energy states which are axisymmetric. In cylindrical geometries, aspect ratios with $L / R_{0}>1.67$, where $L$ is the length and $R_{0}$ is the radius of the flux conserving boundary, the spheromak is tilt unstable. ${ }^{15,16}$ Thus, the Taylor states in oblate geometries $\left(L / R_{0}<1.67\right)$ are axisymmetric and are nonaxisymmetric in prolate geometries $\left(L / R_{0}>1.67\right)$. The flux-conserver used in the experiments described here has $L / R_{0}=3$. This geometry has been characterized previously to have a helical state as its lowest energy eigenstate, in agreement with the WoltjerTaylor theory. ${ }^{17}$

\section{EXPERIMENTAL APPARATUS}

The SSX is a flexible facility for studying magnetic reconnection and spheromak physics. It is equipped with two coaxial magnetized plasma guns for generating spheromaks, one located on either end of the vacuum vessel. A cross section of SSX is shown in Fig. 1. The guns inject spheromaks coaxially into the flux-conserver from either end; the copper flux-conserver defines the flux conserving boundary. The plasmas are confined only by the flux-conserver; the one used in the experiment described here has a length of $L=0.6 \mathrm{~m}$ and a radius $R_{0}=0.2 \mathrm{~m}$. There are no vacuum fields to speak of, other than the small field due to the "stuffing" flux of the plasma guns. SSX plasmas have electron densities up to $10^{21} \mathrm{~m}^{-3}$ and magnetic fields on the order of 0.1 T. Ion and electron temperatures are typically $T_{i} \sim T_{e} \approx 20 \mathrm{eV}$, although $T_{i}$ can go higher when heated by reconnection. ${ }^{18}$ The plasma is fully ionized and magnetized $\left(\rho_{i} \ll R_{0}\right)$ and is in the magnetohydrodynamic (MHD) regime.

Magnetic signals are directly measured by three-axis probes installed in quartz or thin-walled stainless steel tubes. a)
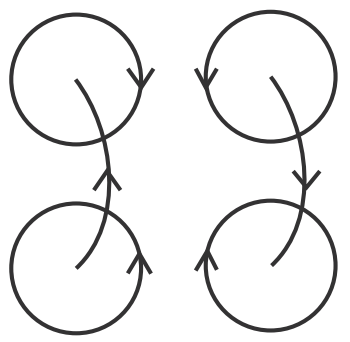

c)

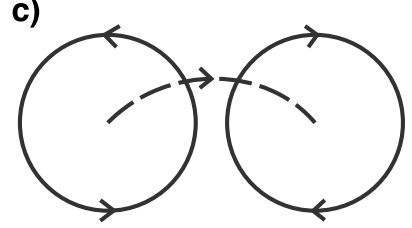

b)

d)

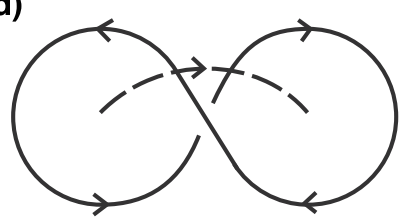

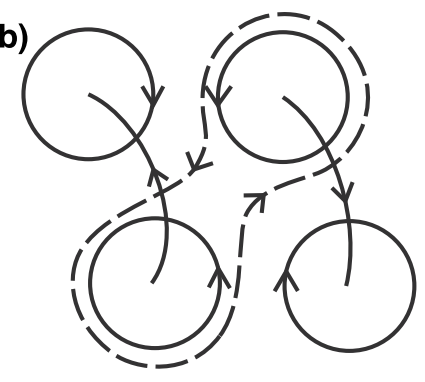

FIG. 2. A cartoon illustrating how the two spheromaks merge. In (a), the initial axisymmetric orientation of the two spheromaks is shown. The magnetic field in the layer of interaction between the two spheromaks is codirected everywhere, with a field null at the center. In (b), the spheromaks start to tilt in the same direction allowing the poloidal fields to now reconnect, shown by the dashed line. In (c), the original poloidal flux has fully reconnected, becoming the toroidal field of the final state. The original toroidal flux reconfigures in $(\mathrm{d})$.

These probe-stalks, containing 288 individual probes, are inserted into the plasma to record the local magnetic field. They are integrated by hardware and then acquired at a rate of $1.25 \mathrm{MHz}$, much faster than any relevant dynamic timescale.

Line averaged ion flow velocities and ion temperatures are measured with a $1.33 \mathrm{~m}$ ion Doppler spectrometer (IDS) ${ }^{19}$ High spectral resolution $(\Delta v=5 \mathrm{~km} / \mathrm{s})$ is achieved with an Echelle grating operating at 25 th order. High temporal resolution $(1 \mu \mathrm{s})$ is achieved by using a 32 channel photomultiplier tube array. The system views a $1 \mathrm{~cm}$ diameter chord across the plasma volume at the axial midplane.

\section{HiFi SIMULATION}

In the simulation described in this paper, an idealized system consisting of two initially identical stationary coaxial uniform $\lambda$ (Bessel function) spheromaks with major radius $R_{0}$ is considered. The spheromaks are placed next to each other to fill a perfectly conducting cylindrical flux-conserver of length $L=3 R_{0}$, such that the radial components of the respective magnetic fields are both negative at the interface and a single interior magnetic null-point $(\boldsymbol{B}=0)$ is formed at the center of the domain [see Fig. 2(a)]. This initial condition is an equilibrium and an eigenstate of Eq. (1) with $\lambda_{i}=4.37 / R_{0}$. Initial density $\rho$ and pressure $p$ are taken to be uniform. The laboratory setup at SSX has similar characteristics; naturally, the experimentally produced spheromaks are neither stationary nor precisely identical when the two magnetic structures begin to interact with each other. Values for $R_{0}=0.2 \mathrm{~m}, B_{0}=0.1 \mathrm{~T}$, and $n_{0}=4 \times 10^{20} \mathrm{~m}^{-3}$ are taken from the SSX experiment as the normalization parameters for the simulation, with $v_{\mathrm{Alf}}=B_{0} /\left(4 \pi n_{0} m_{p}\right)^{1 / 2}=10.9 \mathrm{~cm} / \mu \mathrm{s}$ and $\tau_{\mathrm{Alf}}=R_{0} / v_{\mathrm{Alf}}=1.83 \mu \mathrm{s}$. With ion and electron temperatures on the order of $10-20 \mathrm{eV}$, the plasmas have low thermal to 
magnetic pressure ratio and both ion and electron orbits are well confined by the magnetic field $\left(\rho_{i}<0.5 \mathrm{~cm}\right.$, $\left.\rho_{e} \ll \rho_{i} \ll R_{0}\right)$. These experimental parameters justify the use of the compressible MHD approximation in the simulation described below. We note that for these plasma parameters, the ion inertial scale $\left(c / \omega_{p i}\right) \approx 1 \mathrm{~cm}$ can be comparable to the smallest dimension of the SSX reconnection region, introducing the possibility that Hall MHD effects may begin to play a role in the reconnection dynamics. ${ }^{20}$ While acknowledging that possibility, Hall MHD effects are neglected in the simulation described here, leaving a full 3D Hall MHD treatment of this problem for a future study.

The simulation is conducted with a 3D implicit spectral element HiFi code, ${ }^{9}$ solving the following set of compressible MHD equations:

$$
\begin{aligned}
& \frac{\partial \rho}{\partial t}+\nabla \cdot[\rho \boldsymbol{v}]=0 \\
& \frac{\partial(\rho \boldsymbol{v})}{\partial t}+\nabla \cdot[\rho \boldsymbol{v} \boldsymbol{v}+p \overline{\mathbf{I}}-\mu \nabla \boldsymbol{v}]=\boldsymbol{J} \times \boldsymbol{B} \\
& \boldsymbol{E}=-\frac{\partial \boldsymbol{A}}{\partial t}=-\boldsymbol{v} \times \boldsymbol{B}-\nu \nabla^{2} \boldsymbol{J} \\
& \frac{3}{2} \frac{\partial p}{\partial t}+\nabla \cdot\left[\frac{5}{2} p \boldsymbol{v}-\kappa \nabla T\right]=\boldsymbol{v} \cdot \nabla p+\mu\|\nabla \boldsymbol{v}\|^{2}+\nu\|\nabla \boldsymbol{J}\|^{2},
\end{aligned}
$$

where $p=\rho T$, and $\kappa, \mu$, and $\nu$ are normalized, uniform, and isotropic heat conduction and viscous coefficients for plasma flow $\boldsymbol{v}$ and current density $\boldsymbol{J}$, respectively. The simulation described below was conducted with normalized $\left.\rho\right|_{t=0}=\left.p\right|_{t=0}=1$ and $\{\kappa, \mu, \nu\}=\left\{10^{-1}, 10^{-2}, 5 \times 10^{-6}\right\}$. The form and magnitude of the dissipation operators used in this study are primarily phenomenological, necessary to provide sufficient dissipation in the nonlinear simulation, and are justified only a posteriori by comparison of the simulation results against the experimental measurements. ${ }^{21}$ The following energy-conserving boundary conditions are imposed: heat insulator $\hat{n} \cdot \nabla T=0$, perfect slip plasma flow $\hat{n} \cdot \nabla(\hat{n} \times \boldsymbol{v})=\mathbf{0}$, and perfect slip plasma current $\hat{n} \cdot \nabla \boldsymbol{J}=\mathbf{0}$, where $\hat{n}$ is the unit normal vector at the boundary surface. The resulting variation in total energy over the duration of the simulation is about 1 in $10^{4}$, and $\approx 1 \%$ of the smallest (kinetic) energy component variation.

\section{QUALITATIVE DESCRIPTION OF GLOBAL DYNAMICS}

The 3-to-1 flux-conserver is a tilt-unstable geometry. The calculated lowest energy eigenstate has $\lambda=17.7 \mathrm{~m}^{-1}$ $\left(\lambda R_{0}=3.54\right)$ and is nonaxisymmetric. The final state was experimentally measured to be dominated by the $n=1$ mode and to possess a flat $\lambda$-profile, consistent with the calculated lowest energy eigenstate. ${ }^{17}$ The process of how the initial state consisting of two spheromak transitions to this final state is described here.
The two spheromaks enter the flux-conserver axisymmetrically and establish themselves, as shown in Fig. 2(a). As described earlier, the toroidal fields of the two spheromaks are oppositely directed and the poloidal fields are codirected everywhere in the plane of interaction between the two spheromaks. There is a magnetic field null-point in the center of the volume. The system evolves by the tilting of both spheromaks in the same direction, as shown in Fig. 2(b). The poloidal field of the "top" of one spheromak is now counterdirected with respect to the poloidal field on the "bottom" of the other spheromak, allowing the poloidal field to begin to reconnect at the central field null. The spheromaks continue to tilt almost a full $90^{\circ}$ as the reconnection proceeds. The reconnecting poloidal fields become the toroidal field of the final nonaxisymmetric state [the view is rotated $90^{\circ}$ in Figs. 2(c) and 2(d) with respect to Figs. 2(a) and 2(b)]. Topologically, once the poloidal flux has completely reconnected, the merging is finished. However, the original toroidal fluxes of the two spheromaks reconfigure by component reconnection ${ }^{22}$ and become the helically twisted poloidal field of the final state, shown in Fig. 2(d). This twisted figure- 8 structure is a rational surface and is evident in the simulation data.

There is good correspondence between the experimental data obtained during the merging process and the HiFi simulation. Both experimental and simulated spheromaks are unstable to the previously described aligned tilting and follow the progression outlined above. The experimentally produced spheromaks are neither stationary nor precisely identical upon injection, although there is a brief phase before merging commences where two volumes of private flux are clearly observed. On the other hand, the initial state in the simulation is an eigenstate of Eq. (1) perturbed by $\delta v_{z}$ $\propto 10^{-2} v_{\text {Alf }}\left(r / R_{0}\right) \sin \left(\pi z / L_{0}\right)$. The angular phase of the tilt is arbitrary, thus the phase of the $\delta v_{z}$ tilting perturbation applied in the simulation was chosen to best correspond with the available experimental data. The tilting progression observed in the simulation is shown in Fig. 3.

A comparison between the SSX experimental and HiFi simulation $\boldsymbol{B}$-field data in the midst of the tilting, reconnection, and relaxation activity is shown in Fig. 4. The SSX measurements are taken in three planes across the cylindrical flux-conserver along four probe-stalks separated angularly by $\Delta \theta=90^{\circ}$ at eight radial locations on each probe-stalk. HiFi simulation data are shown in the equivalent planes through the computation volume. Remarkable similarity in the direction and relative magnitude of the measured and computed $\boldsymbol{B}$-fields is apparent throughout the volume. In particular, the center plane $\theta=315^{\circ}$ probe in SSX and the corresponding $\mathrm{HiFi}$ simulation planes both show that the $\boldsymbol{B}=0$ location has moved out radially from the center of the domain.

\section{NULL-POINT EVOLUTION}

An important feature observed in both the HiFi simulation and the experiment is the three-dimensional nature of the reconnection. As the spheromaks tilt from their initial axisymmetric configuration, the magnetic field null located 

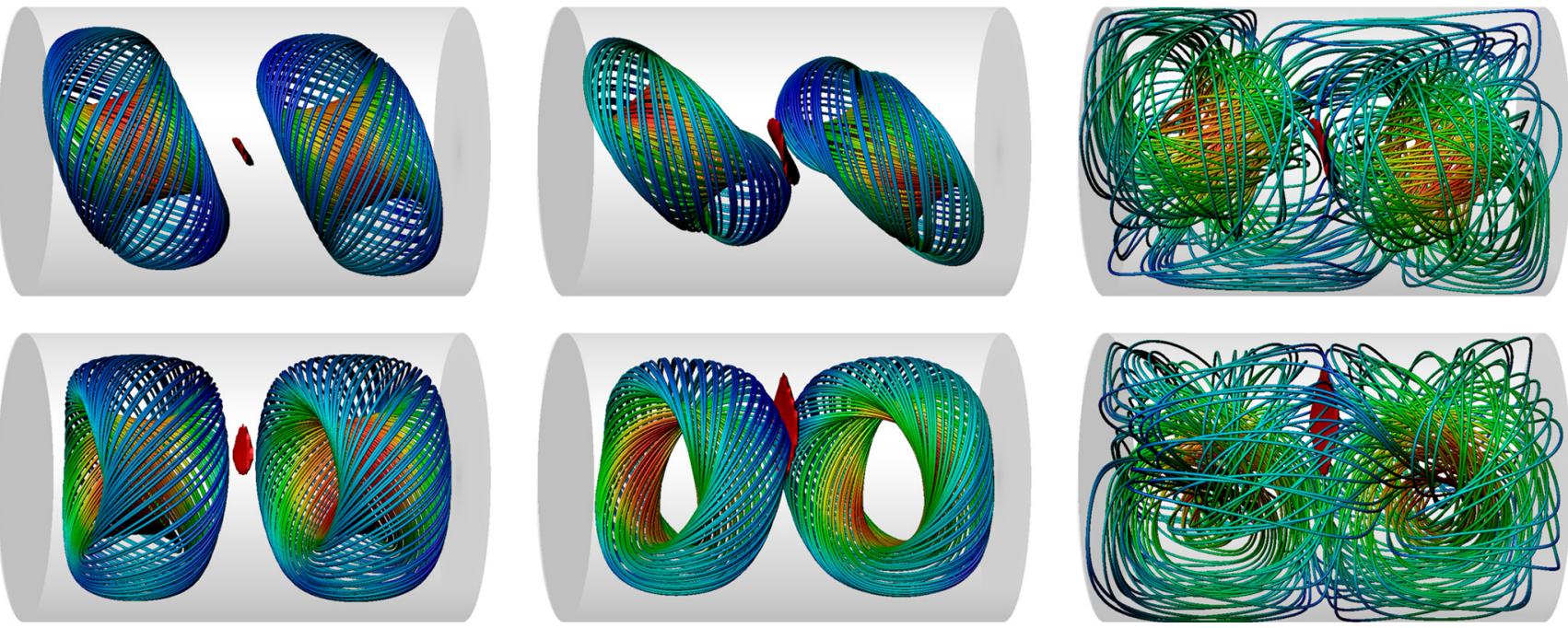

FIG. 3. (Color online) Magnetic field lines from the HiFi simulation, illustrating the two spheromaks, are shown here. Peak in $|\boldsymbol{J}|$ is represented by the surface in the center of the volume. The top row and bottom row both show the same point in time; the images in the bottom row are an orthogonal view to the ones in the top row. Starting at the left, the spheromaks have already begun to tilt at this point in the simulation. As time advances, the spheromaks tilt progressively more, shown in the middle two images, and begin to reconnect, as shown in the images on the right. It is important to note that reconnection is already occurring in the images on the left and continues to occur after the images on the right.

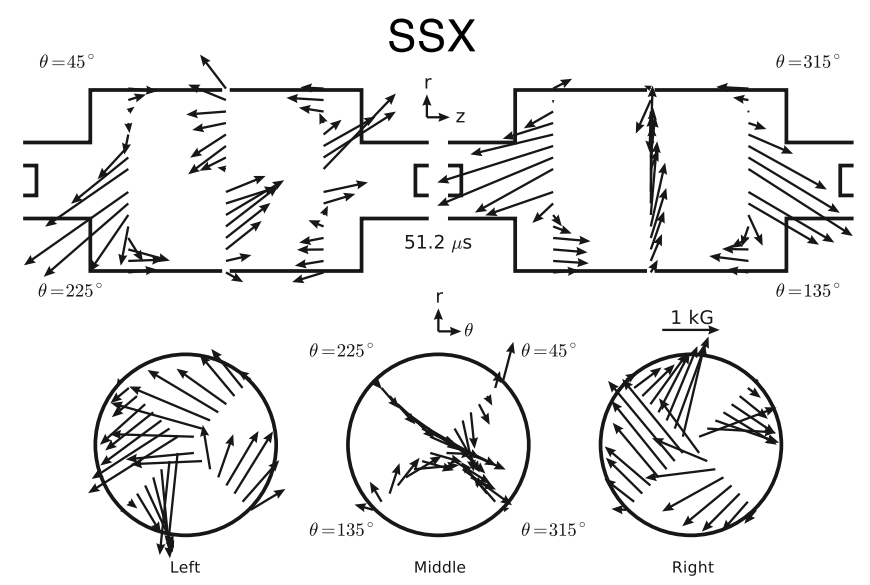

$\mathrm{HiFi}$
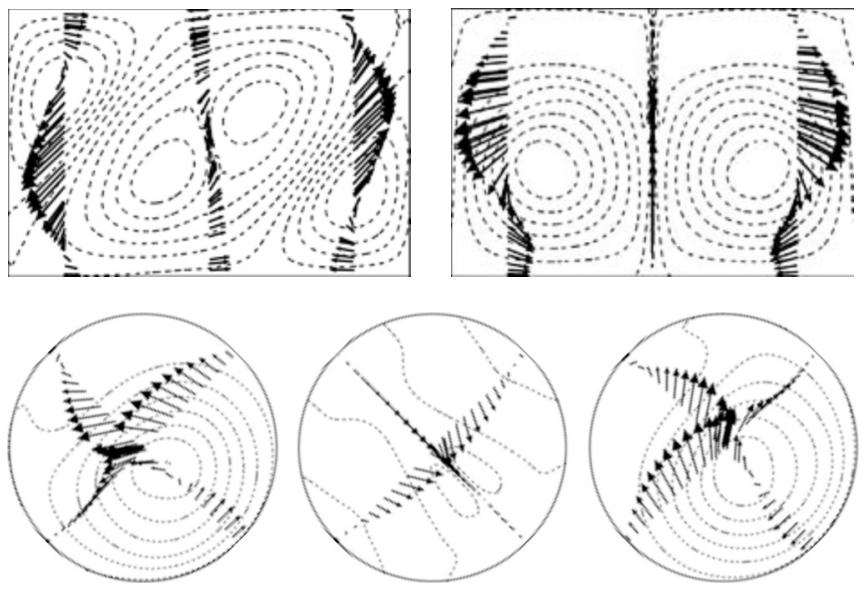

FIG. 4. Comparison of $\boldsymbol{B}$-field measurements in equivalent planes from the SSX experiment (top) and the HiFi simulation (bottom) during the fast relaxation through null-point magnetic reconnection. Note the magnetic null at the bottom-right of the $(\hat{r}, \hat{\theta})$ center frame and top-center of the $(\hat{r}, \hat{z}) \theta$ $=\left\{135^{\circ}, 315^{\circ}\right\}$ frame. The contours in the HiFi data represent $\boldsymbol{B}_{\perp}$. at the center of the volume moves radially outward, parallel to the axes around which the spheromaks are tilting. The radial motion of the field null is caused by the magnetic fields of the tilting spheromaks convecting into the interface plane between them, originally consisting of radially inward directed $\boldsymbol{B}$ everywhere. The motion of the field null can be seen in Fig. 3 by looking at the surfaces representing peak $|\boldsymbol{J}|$, associated here with magnetic reconnection. While reconnection is peaked around the radially moving magnetic field null, there is component reconnection all along the diameter on which the field null travels.

The peak normalized magnitude of the reconnection $\boldsymbol{E}$-field, $\boldsymbol{E}_{\text {rec }} \equiv \boldsymbol{E}_{\text {tot }}+\boldsymbol{v} \times \boldsymbol{B}$, and the ratio of the system's magnetic energy to helicity, $\langle\lambda\rangle \equiv 2 W_{\text {mag }} / K$, throughout the simulation are shown in Fig. 5(a). $\langle\lambda\rangle$ is used for any $\boldsymbol{B}$-field configuration to denote its relaxed state. The motion of the unique magnetic null and of the primary reconnection region during the period of most intense reconnection is shown in Fig. 5(b). It is clear that the motions of the null-point and the reconnection region are coupled as long as the magnetic null remains within the bulk of the plasma, and the fastest $\boldsymbol{B}$-field relaxation, as measured by the change in $\langle\lambda\rangle$, takes place through null-point reconnection.

In the experiment, the initial configuration of the interaction region is well matched to the initial conditions in the simulation. The magnetic field null is present roughly at the center of the flux-conserver, as in the simulation. Shortly after the tilting commences, the field null moves radially outward along one of the midplane probes for the duration of the merging. $|\boldsymbol{B}|$ along the probe is shown at two different times during the same shot in Fig. 6. The field null-point is clearly visible at $r=12.7 \mathrm{~cm}$ at $t=52.8 \mu \mathrm{s}$. Slightly later in time, at $t=57.6 \mu \mathrm{s}$, as the reconnection continues, the field null-point has moved out radially to $r=15.2 \mathrm{~cm}$, corresponding to a null-point velocity of $\approx 5 \mathrm{~km} / \mathrm{s}$. (Note that the last probe position, at $r=20.3 \mathrm{~cm}$, is located at the flux- 


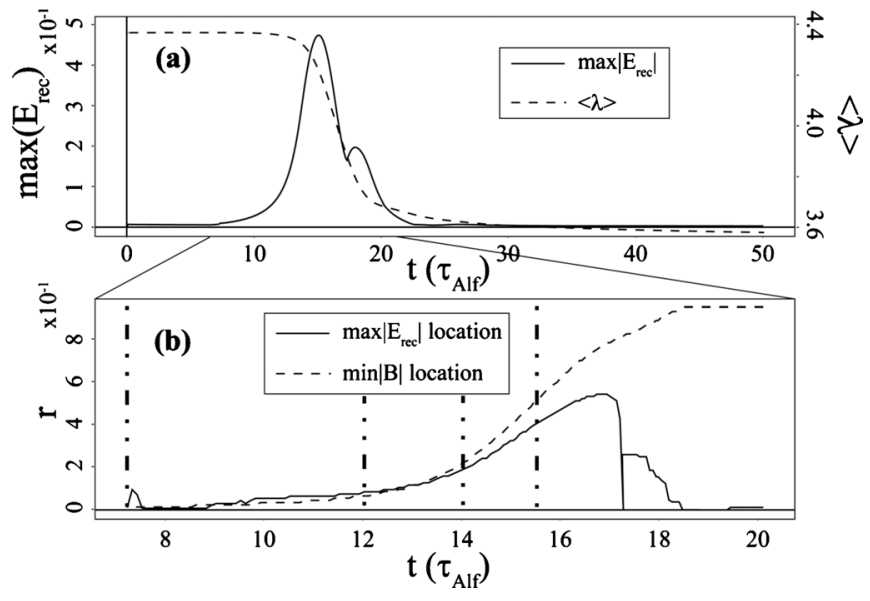

FIG. 5. Time-traces from the simulation of (a) reconnection $\boldsymbol{E}$-field and $\langle\lambda\rangle$ over the duration of the simulation (zero is suppressed on the $\langle\lambda\rangle$ axis) and (b) radial location of the primary reconnection region and the $\boldsymbol{B}$-field null during the most intense reconnection (zoomed-in time-scale). The reconnection region is observed to track the magnetic null through the bulk of the plasma. Markers in panel (b) denote times for which the structure of the null is shown in Fig. 7.

conserver wall in the midplane diagnostic gap, which explains its low values of $|\boldsymbol{B}|$ at both points in time.) At later times, the null-point moves to larger $r$ until it has left the flux-conserver volume.

Figure 7 shows the evolution of the structure of the simulated magnetic fields surrounding the null-point, and therefore also the reconnection region, as the magnetic null is moving radially through the plasma. Early in time, while the null remains near the cylindrical axis of the domain [Figs. 7(a) and 7(b)], the spine and the fan of the null-point ${ }^{23}$ can be easily identified. However, as the null-point moves out to larger radius later in time [Figs. 7(c) and 7(d)], the spine and the fan, while still distinct, begin to gradually merge. This type of spine-fan reconnection has been previously observed in MHD simulations of driven null-point reconnection enforced by line-tied flows in the proximity of a prescribed diffusive region. ${ }^{23,24}$ Yet, due to the nature of the previously considered configurations, a critical element in the dynamics of spine-fan reconnection, the possibility of the motion of the null-point itself, has been missing. The radial

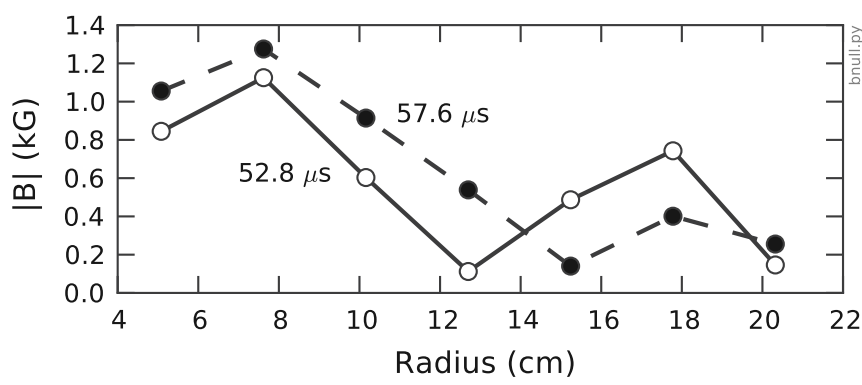

FIG. 6. Radial profiles of $|\boldsymbol{B}|$ from the experiment at two times during the same shot, 52.8 and $57.6 \mu \mathrm{s}$. These two times are during the merging phase. The field null-point is clearly visible at $r=12.7 \mathrm{~cm}$ at $t=52.8 \mu \mathrm{s}$. Slightly later in time, at $t=57.6 \mu \mathrm{s}$, as the reconnection continues, the field nullpoint has moved out radially to $r=15.2 \mathrm{~cm}$. The flux-conserver is located at $20 \mathrm{~cm}$. The last probe is located at the flux-conserver wall in the diagnostic gap, causing the small values of $|\boldsymbol{B}|$ at this location.

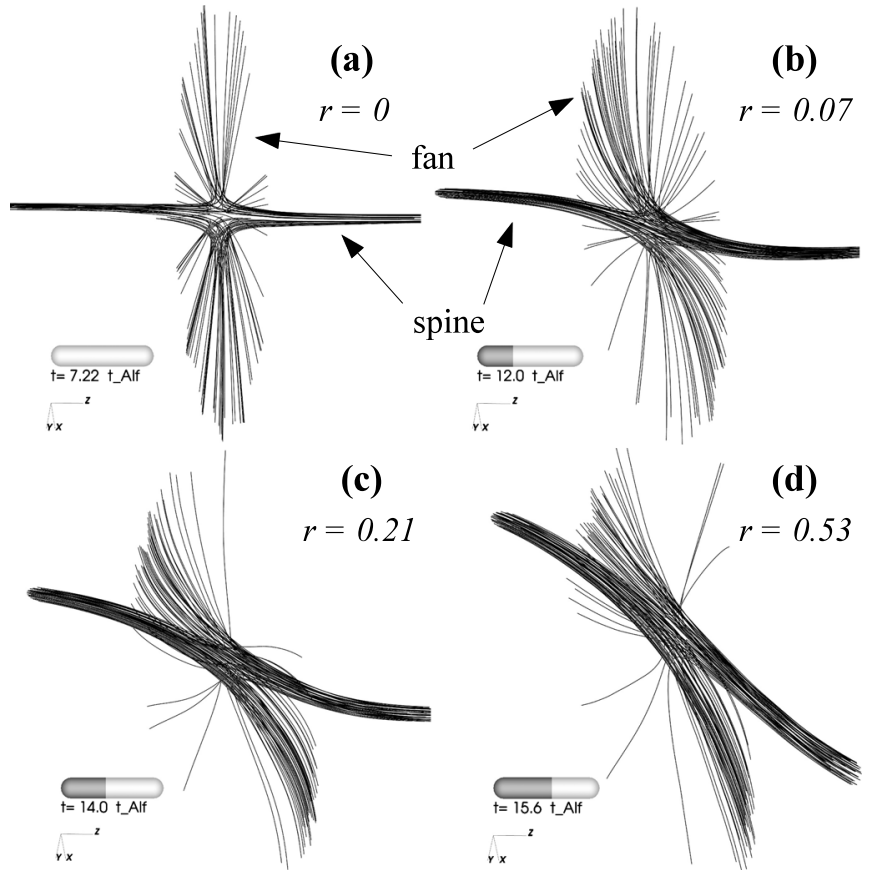

FIG. 7. Evolving structure of field lines from the simulation around the null-point, as it is radially moving through the domain. Streamlines of $\boldsymbol{B}$-field originate on a small sphere surrounding and tracking the null-point, showing the dynamics of spine-fan reconnection.

trajectory of the null-point shown in Fig. 5(b) implies its velocity to be up to $\approx 0.2 v_{\text {Alf }}$, which is a significant fraction of the maximum reconnection outflow velocity measured to be $\approx 1.1 v_{\text {Alf }}$.

While null-point reconnection is dominant, Fig. 5 also shows that reconnection does not cease when the magnetic null leaves the bulk of the plasma. A secondary peak in the reconnection rate is observed at $t \approx 18 \tau_{\text {Alf }}$ and represents component reconnection that continues to enable relaxation toward the helical Taylor state characterized by $\lambda_{f}=3.54 / R_{0}{ }^{17}$

In addition to the similar magnetic field structure, Alfvénic outflows emanating from the reconnection region are observed in both the simulation and the experiment. ${ }^{25}$ Inspection of the flows in the simulation reveals that the flows are largest in magnitude around the field null-point, with $v=v_{\text {Alf }}$ corresponding to $\boldsymbol{B}=0.1 \mathrm{~T}$. This is illustrated in Fig. 8. The outflow follows the field null as it moves radially outward.

IDS measurements made during the cohelicity merging experiments also reveal two component flows which rapidly vary with time during the merging phase (Fig. 9). Outflows are not observed on every plasma discharge due to the small field of view of the IDS instrument coupled with the arbitrary phase of spheromak tilting about the axis. When observed, the peak magnitude of the outflows is $25-30 \mathrm{~km} / \mathrm{s}$, both toward and away from the spectrometer. These flows, if Alfvénic, correspond to $\boldsymbol{B}$-fields of $200-300 \mathrm{G}$ in the reconnection layer. Conversely, the flows are $\approx 25 \%$ of $v_{\text {Alf }}$ if correlated with the $\boldsymbol{B}$-fields of the bulk plasma, where $\boldsymbol{B} \approx 0.1 \mathrm{~T}$. This is somewhat at odds with what is seen in the simulation, with the difference likely related to the micro- 


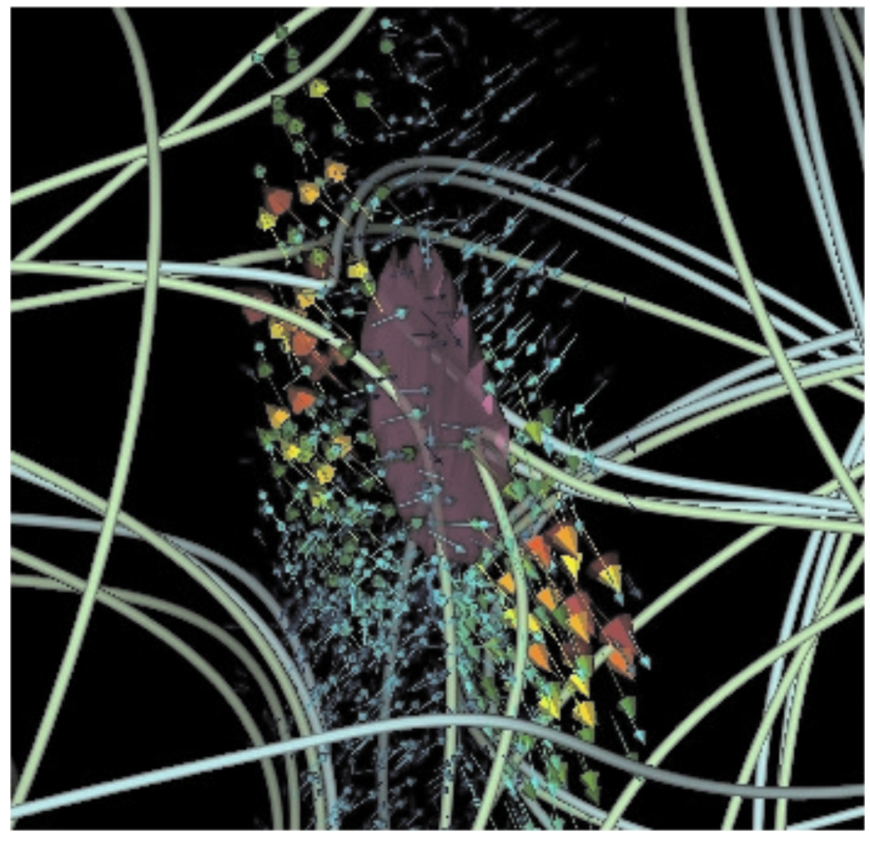

FIG. 8. (Color online) Image of the simulated 3D reconnection region during the most intense reconnection $\left(t=14.987 \tau_{\mathrm{Alf}}\right)$. Streamlines show the magnetic field, arrows show jets of Alfvénic plasma outflow $\left(v_{\max } \approx v_{\mathrm{Alf}}\right)$, and the contour shows the region of peaked $\boldsymbol{J}$.

scopic description of the reconnection region in the simulation. These flows are only observed during the merging phase of the plasma. Once the merging is complete, the twocomponent flows subside and little or no velocity shift is observed for the duration of the plasma.

It should be noted that the IDS data were obtained in a different flux-conserver geometry, with $L=0.4 \mathrm{~m}$ and a radius $R_{0}=0.2 \mathrm{~m}$. The IDS diagnostic was upgraded after the original experiments in the $L=0.6 \mathrm{~m}$ flux-conserver, provid-

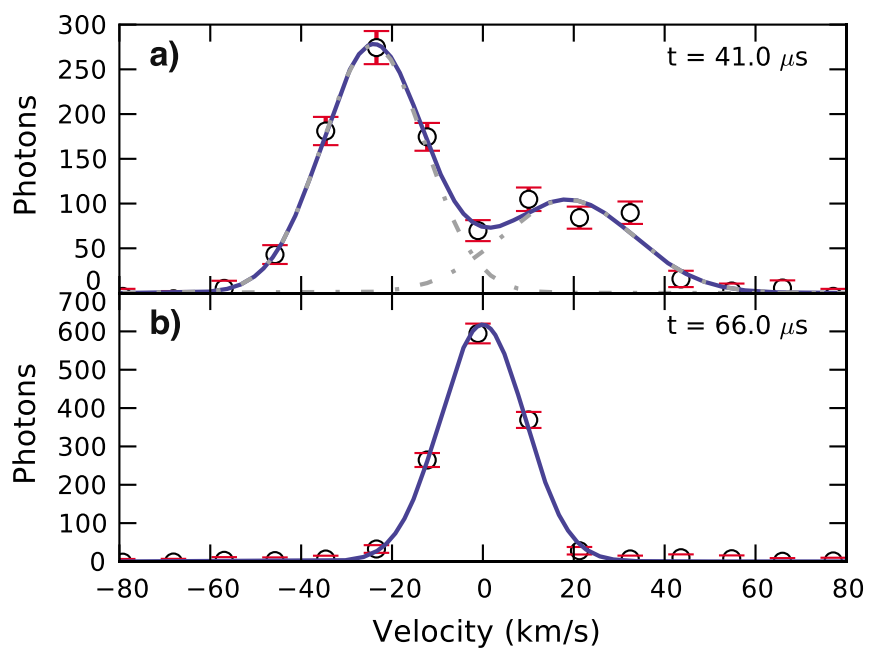

FIG. 9. (Color online) Ion Doppler spectrometer measurements. During the merging [shown in (a)], two component flows appear, one moving toward the spectrometer and one moving away, at approximately $\pm 25 \mathrm{~km} / \mathrm{s}$. The magnitude of the velocity shifts and the total photon counts of the two peaks change rapidly during the merging. After merging is completed (b), the two component structure disappears. Gaussian fits are indicated by the solid line. Data are from a single shot.
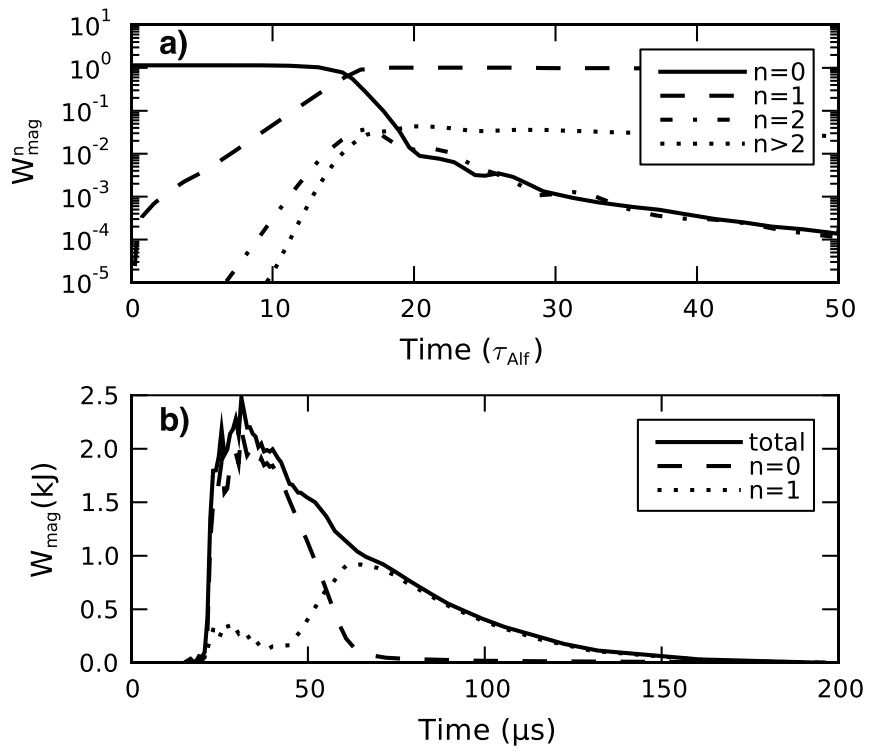

FIG. 10. The top plot (a), from the simulation, shows the time evolution of magnetic energy in cylindrical Fourier modes $n=0,1,2$ and $n>2$ integrated over the full cylindrical domain $(r, z) \in\left[0, R_{0}\right] \times\left[-1.5 R_{0}, 1.5 R_{0}\right]$. The bottom plot (b), from the experiment, shows the total magnetic energy and the $n=0,1$ components. The plasma has reached its relaxed state at $75 \mu \mathrm{s}$.

ing higher quality data. However, the move to the smaller flux-conserver precluded the use of magnetic probes with the same extensive coverage as in the $L=0.6 \mathrm{~m}$ experiments. Simulations of the two geometries with the HiFi code show substantially the same behavior.

\section{DISCUSSION}

The dynamics of the simulated magnetic relaxation are further quantified in Fig. 10(a), where the spectral magnetic energy content in $n=0,1,2$ and the sum over $n>2$ cylindrical Fourier modes, $W_{\text {mag }}^{n}$, are shown as a function of time. Here, the linear growth of the tilting instability followed by rapid transition from the axisymmetric $n=0$ to the helical $n=1$ state becomes apparent. Despite the complexity of localized 3D reconnection that allows for such fast relaxation, the $n \geq 2$ modes account for under $2 \%$ of total magnetic energy throughout the simulation, i.e., there is no evidence of significant magnetic fluctuations at higher mode numbers. It is also worth noting that unlike the $n=0,2$ modes, the total sum of magnetic energy in the $n>2$ modes generated during reconnection appears to be subject only to the overall resistive decay rather than relaxation to the lowest energy $n=1$ eigenstate.

The time evolution of the experimentally measured magnetic energy is also shown in Fig. 10(b). The magnetized plasma enters the volume at $20 \mu \mathrm{s}$ and is primarily axisymmetric as can be seen by the large $n=0$ component until $45 \mu \mathrm{s}$. Over the course of 20-25 $\mu \mathrm{s}$, most of the magnetic energy switches from the $n=0$ to the $n=1$ mode with little dissipation. This is the period when the two initial spheromaks are tilting and merging. The plasma reaches its final relaxed state after $75 \mu \mathrm{s}$. Like the simulation, there is no evidence for broad-scale magnetic fluctuations and no large 
scale turbulence is seen. Relaxation to the final state occurs primarily through the dynamical activity occurring at the radially moving null-point.

Accounting for the helicity and energy input at the beginning of the discharge and the helicity and energy content of the relaxed state shows whether or not helicity is conserved during the relaxation of the plasma to its minimum energy eigenstate. Helicity injected by the coaxial plasma gun is given by

$$
K_{\text {inj }}=\int 2 V \Phi d t
$$

where $V$ is the bias voltage on the gun and $\Phi$ is the stuffing flux. Energy injected by the gun is given by

$$
W_{\text {inj }}=\int V I d t
$$

where $I$ is the gun current. Helicity contained in the relaxed plasma is related to the magnetic stored energy of the plasma once relaxed, $W_{\text {relax }}$, and the $\lambda_{0}$ of the relaxed state by Eq. (4). $\lambda_{0}$ is calculated in Ref. 17. $W_{\text {mag }}$ can be calculated by summing the square of the magnetic probe data and integrating over the volume of the plasma,

$$
W_{\text {mag }}=\frac{1}{2 \mu_{0}} \int B^{2} d V \text {. }
$$

Evaluating it at the time when the plasma is fully relaxed gives us $W_{\text {relax }}$. Since the probes sample the plasma volume at 96 discreet locations, the volume is divided into $96 \mathrm{seg}$ ments for this calculation, each segment centered on a probe.

Energy accounting of the experiment shows that the energy injected by the two plasma guns is $W_{\mathrm{inj}} \approx 2800 \mathrm{~J}$, while the peak measured magnetic energy of the plasma before it relaxes is $W_{\max } \approx 2500 \mathrm{~J}$ [Fig. 10(b)]. This difference is expected as some of the energy injected from the guns goes into kinetic energy of the plasma. The magnetic energy of the plasma when it first reaches its relaxed state at $75 \mu$ s is $W_{\text {relax }} \approx 850 \mathrm{~J}$ [Fig. 10(b)]. Thus, the ratio of initial magnetic energy to the peak magnetic energy of the relaxed state is $W_{\text {max }} / W_{\text {relax }} \approx 3$. This is consistent with earlier research that shows the drop in magnetic energy is proportional to the ratio of the gun $\lambda_{g}$ to the $\lambda_{0}$ of the relaxed state. ${ }^{26}$ In SSX, $\lambda_{g}=48 \mathrm{~m}^{-1}$ and $\lambda_{0}=17.7 \mathrm{~m}^{-1}$ for the relaxed state, giving a ratio of $\lambda_{g} / \lambda_{0}=2.7$. Helicity accounting shows that the total injected helicity from both guns is $K_{\mathrm{inj}}=0.99 \times 10^{-4} \mathrm{~Wb}^{2}$ while the measured peak helicity of the final relaxed state $K_{\text {relax }}=1.2 \times 10^{-4} \mathrm{~Wb}^{2}$. Thus, helicity is conserved during the relaxation process in accord to Taylor relaxation. The $20 \%$ discrepancy can possibly be explained by the deviations from flatness of the $\lambda$-profile as well as error in the helicity content calculation of the final state introduced by limited volumetric coverage of the magnetic probes of the plasma volume.

Considering the mismatch in precise initial and boundary conditions, as well as the ad hoc form of the numerical magnetic diffusion operator, there is good agreement between the dynamical features of 3D magnetic reconnection and relaxation demonstrated in the experiment and the simu- lation. As inferred from Figs. 5 and 10, the time-scale $t_{R}$ of the global magnetic field relaxation observed in the simulation is $t_{R} \approx 10-15 \tau_{\mathrm{Alf}} \approx 18-27 \mu s$. This is in agreement with the experimentally observed relaxation time-scales of 20-25 $\mu \mathrm{s}$. Furthermore, in both the experiment and the simulation, the velocity of the magnetic null-point corresponds to $\approx 0.2$ of the reconnection outflow velocity, i.e., local upstream Alfvén velocity, underlining the importance of the nonsteady nature of the moving globally threedimensional magnetic reconnection site.

It should be emphasized that the numerical simulation has been converged in both spatial and temporal resolutions with respect to the results presented above. Additionally, a similar computational study conducted by an independent group using entirely different numerical methods, as well as different boundary conditions and diffusion operators, has demonstrated qualitative agreement with the conclusions of this work. ${ }^{27}$ This latter fact suggests that the $3 \mathrm{D}$ magnetic relaxation is fundamentally governed by the properties of ideal MHD and may be insensitive to the specific form of, although necessarily requires some, magnetic dissipation.

In summary, this paper describes a joint simulationexperiment study of the cohelicity merging of two spheromaks. Relaxation of the plasma to the lowest energy eigenstate occurs through reconnection primarily at one field nullpoint, without evidence of significant magnetic fluctuations occurring at high mode numbers. Both the global magnetic fields and the dynamical time-scales are shown to be in good agreement between the experiment and the simulation. The structure of the magnetic null is characterized and the null itself is shown to move, achieving velocities up to $0.2 v_{\mathrm{Alf}}$. It is further demonstrated that the motion of the magnetic null is correlated with that of the primary reconnection region and greater implications of a rapidly moving reconnection region are discussed. Finally, the dynamics of relaxation facilitated by $3 \mathrm{D}$ reconnection is quantified, showing rapid transfer of energy from the initially axisymmetric to a dominantly helical state, demonstrating general agreement with the WoltjerTaylor relaxation theory.

\section{ACKNOWLEDGMENTS}

This work was supported by the Department of Energy and the National Science Foundation. We gratefully acknowledge the contributions of A. H. Glasser and W. Lowrie to the development of the HiFi code. We also thank M. G. Linton, M. J. Schaffer, E. V. Belova, and C. E. Myers for helpful discussions. Additionally, the authors would like to acknowledge partial support from the Office of Naval Research for the work presented in the manuscript.

${ }^{1}$ T. R. Jarboe, Plasma Phys. Controlled Fusion 36, 945 (1994).

${ }^{2}$ T. R. Jarboe, I. Henins, H. W. Hoida, R. K. Linford, J. Marshall, D. A. Platts, and A. R. Sherwood, Phys. Rev. Lett. 45, 1264 (1980).

${ }^{3}$ A. al-Karkhy, P. K. Browning, G. Cunningham, S. J. Gee, and M. G. Rusbridge, Phys. Rev. Lett. 70, 1814 (1993).

${ }^{4}$ C. G. R. Geddes, T. W. Kornack, and M. R. Brown, Phys. Plasmas 5, 1027 (1998).

${ }^{5}$ S. Woodruff, B. W. Stallard, H. S. McLean, E. B. Hooper, R. Bulmer, B. I. Cohen, D. N. Hill, C. T. Holcomb, J. Moller, and R. D. Wood, Phys. Rev. Lett. 93, 205002 (2004). 
${ }^{6}$ Y. Ono, M. Yamada, T. Akao, T. Tajima, and R. Matsumoto, Phys. Rev. Lett. 76, 3328 (1996).

${ }^{7}$ M. R. Brown, Phys. Plasmas 6, 1717 (1999).

${ }^{8}$ C. D. Cothran, A. Falk, A. Fefferman, M. Landreman, M. R. Brown, and M. J. Schaffer, Phys. Plasmas 10, 1748 (2003).

${ }^{9}$ V. S. Lukin, Ph.D. thesis, Princeton University, 2007.

${ }^{10}$ M. Yamada, Y. Ono, A. Hayakawa, M. Katsurai, and F. W. Perkins, Phys. Rev. Lett. 65, 721 (1990).

${ }^{11}$ E. G. Zweibel and M. Yamada, Annu. Rev. Astron. Astrophys. 47, 291 (2009).

12 J. B. Taylor, Phys. Rev. Lett. 33, 1139 (1974).

${ }^{13}$ J. B. Taylor, Rev. Mod. Phys. 58, 741 (1986).

${ }^{14}$ L. Woltjer, Proc. Natl. Acad. Sci. U.S.A. 44, 489 (1958).

${ }^{15}$ A. Bondeson, G. Marklin, Z. G. An, H. H. Chen, Y. C. Lee, and C. S. Liu, Phys. Fluids 24, 1682 (1981).

${ }^{16}$ J. M. Finn, W. M. Manheimer, and E. Ott, Phys. Fluids 24, 1336 (1981).

${ }^{17}$ C. D. Cothran, M. R. Brown, T. Gray, M. J. Schaffer, and G. Marklin, Phys. Rev. Lett. 103, 215002 (2009).

${ }^{18}$ V. H. Chaplin, M. R. Brown, D. H. Cohen, T. Gray, and C. D. Cothran, Phys. Plasmas 16, 042505 (2009).

${ }^{19}$ C. D. Cothran, J. Fung, M. R. Brown, and M. J. Schaffer, Rev. Sci. Instrum. 77, 063504 (2006).
${ }^{20}$ J. Birn, J. Drake, M. Shay, B. Rogers, R. Denton, M. Hesse, M. Kuznetsova, Z. Ma, A. Bhattacharjee, A. Otto, and P. Pritchett, J. Geophys. Res., [Space Phys.] 106, 3715, doi:10.1029/1999JA900449 (2001).

${ }^{21}$ We note that instead of the usual resistive term $\eta \boldsymbol{J}$, magnetic dissipation is introduced in Eq. (7) via the viscous, often referred to as hyper-resistive, term $\nu \nabla^{2} \boldsymbol{J}$. This choice is made to accommodate the desired boundary conditions: to allow for nonzero tangential current density $(\hat{n} \times \boldsymbol{J} \neq \mathbf{0})$ at a perfectly conducting $(\hat{n} \times \boldsymbol{E}=\mathbf{0})$ nonpenetrable $(\hat{n} \cdot \boldsymbol{v}=0)$ flux-conserver wall, while also satisfying Eq. (7). The resulting effective magnetic Lundquist number $S^{\mathrm{eff}} \equiv \delta^{2} /\left(\nu R_{0}^{2}\right)=500$ for the reconnection region of width $\delta \approx 1 \mathrm{~cm}=R_{0} / 20$ is consistent with the experimental value of $S \approx 10^{3}$.

${ }^{22}$ Component reconnection, or guide field reconnection, occurs when the reconnecting magnetic fields are not fully antiparallel. Only the opposing components of the fields reconnect.

${ }^{23}$ E. R. Priest and D. I. Pontin, Phys. Plasmas 16, 122101 (2009).

${ }^{24}$ D. I. Pontin, A. Bhattacharjee, and K. Galsgaard, Phys. Plasmas 14, 052106 (2007).

${ }^{25}$ M. R. Brown, C. D. Cothran, and J. Fung, Phys. Plasmas 13, 056503 (2006).

${ }^{26}$ C. W. Barnes, J. C. Fernandez, I. Henins, H. W. Hoida, T. R. Jarboe, S. O. Knox, G. J. Marklin, and K. F. McKenna, Phys. Fluids 29, 3415 (1986).

${ }^{27}$ C. E. Myers and E. Belova, Bull. Am. Phys. Soc. 54, UP8.00018 (2009). 